\title{
Avaliação da função pulmonar e força muscular respiratória de dependentes químicos em tratamento
}

\section{Evaluation of pulmonary function and respiratory muscular strength of chemical dependents in treatment}

\author{
Patrícia Cesar Nascimento Peres'; Meirieli Jeane Cian; Tamiris Rodrigues Grandi'; Mateus Dias \\ Antunes'; Sônia Maria Marques Gomes Bertolini1,2 \\ 1 Centro Universitário de Maringá - UNICESUMAR. Maringá, PR - Brasil \\ 2 Instituto Cesumar de Ciência, Tecnologia e Inovação - ICETI. Maringá, PR - Brasil.
}

Endereço para Correspondência:

Mateus Dias Antunes

Av. Guedner, 1610 - Jardim Aclimação,

87050-390 - Maringá - PR [Brasil]

mateus_antunes03@hotmail.com

\begin{abstract}
Resumo
Introdução: anualmente vem aumentando o número do uso do crack na população brasileira. Objetivo: caracterizar o perfil da população em tratamento no Centro de Atenção Psicossocial para Álcool e outras Drogas (CAPS/AD), para uso do crack na cidade de Maringá e avaliar a função pulmonar e força muscular respiratória desses indivíduos. Método: trata-se de uma pesquisa transversal, quantitativa e observacional com 15 indivíduos dependentes de crack em tratamento. Foram avaliados a presença de sinais e sintomas respiratórios e hábitos pregressos, a função pulmonar e a força da musculatura respiratória. Resultados: dentre os resultados encontrados, $60 \%$ dos indivíduos não apresentaram distúrbios ventilatórios, 13,3\% tiveram CVF adequado, 40,0\% VEF1 adequado e o mesmo percentual com o IT também adequado. 66,7\% tiveram PI máx e PE máx adequadas. Conclusão: os resultados obtidos com a realização desse trabalho não apresentaram alterações significativas para a função pulmonar e para a força muscular respiratória da maioria dos usuários em tratamento no CAPS/AD.
\end{abstract}

Descritores: Cocaína Crack; Usuários de drogas; Espirometria; Fisioterapia; Promoção da Saúde.

\begin{abstract}
Introduction: the number of crack use in the Brazilian population has increased annually. Objective: to characterize the profile of the population being treated at the Psychosocial Care Center for Alcohol and Other Drugs (CAPS /AD), for crack use in the city of Maringá and to evaluate the pulmonary function and respiratory muscle strength of these individuals. Method: This is a cross-sectional, quantitative and observational study with 15 crack-dependent individuals under treatment. The presence of respiratory signs and symptoms and previous habits, pulmonary function and respiratory muscle strength were evaluated. Results: Among the results found, $60 \%$ of the individuals did not present ventilatory disorders, $13.3 \%$ had adequate FVC, $40.0 \%$ had adequate FEV1, and the same percentage had adequate IT. $66.7 \%$ had adequate PI max and PE max. Conclusion: the results obtained with this study did not present significant alterations for pulmonary function and respiratory muscle strength of the majority of users under treatment in CAPS/AD.
\end{abstract}

Keywords: Crack Cocaine; Drug users; Spirometry; Physical Therapy Specialty; Health Promotion. 


\section{Introdução}

O crack chegou ao Brasil por volta da década de 1980 e nos dias atuais é considerada a droga mais consumida em grandes centros urbanos como São Paulo. Esse aumento do consumo de crack contribui para problemas de saúde pública e segurança ${ }^{1-5}$. A droga é denominada como a "droga da morte", considerada de fácil uso, onde os usuários fumam em cachimbos que são produzidos sem dificuldades por eles mesmos, através de recipientes de plásticos e latinhas. Seus efeitos devastadores levam a quadros sérios e preocupantes ${ }^{6}$.

Os sintomas respiratórios estão presentes em 25 a 60\% dos usuários de crack, no entanto poucos procuram assistência médica ${ }^{3}$. Por ser um órgão exposto à combustão do crack, os pulmões e a respiração passam por alguns sintomas respiratórios agudos como dispneia, tosse, dor torácica, expectoração com resíduos de carbono, hemoptise, barotrauma, edema pulmonar, exacerbação da asma, fenômenos de hipersensibilidade e deterioração da função pulmonar².

Atualmente o consumo de crack tem uma alta prevalência no Brasil ${ }^{7}$ o que faz com que esse número de usuário aumente ainda mais, já que é uma droga de fácil acesso, gerando consequências individuais, familiares e sociais ${ }^{8}$.

Nesse sentido, este estudo surgiu da necessidade de conhecer quem são estes usuários e como está a sua saúde respiratória para que possamos propor ações de promoção da saúde, além disso, para buscar alternativas de serviços que possam melhorar a adesão terapêutica e de autocuidado para recuperação da qualidade de vida dessa população.

Mediante aumento do uso do crack pela população brasileira e sabendo que acarreta inúmeras complicações na saúde dos usuários, o presente estudo teve como objetivos caracterizar o perfil da população em tratamento no Centro de Atenção Psicossocial para Álcool e outras Drogas (CAPS/AD), para o uso do crack na cidade de Maringá e avaliar a função pulmonar e força muscular respiratória desses indivíduos.

\section{Material e métodos}

Estudo transversal de natureza quantitativa, realizado no CAPS/AD, localizado na cidade de Maringá, PR. Foram incluídos nesse estudo os dependentes químicos usuários de crack em tratamento, e excluídos indivíduos do sexo feminino, comprometimento cognitivo e não adesão ao tratamento.

Após aprovação do Comitê de Ética e Pesquisa com Seres Humanos conforme parecer $n^{\circ} 1.684 .195$ os indivíduos foram inicialmente orientados sobre a importância do projeto e foram sugeridos a assinarem o Termo de Consentimento Livre e Esclarecido.

Após, foram submetidos à avaliação antropométrica e preenchimento de ficha de avaliação fisioterapêutica, com análise de antecedentes pulmonares. Na sequência foi realizada a espirometria (Espirômetro Spirostik Complete - Geratherm), o teste foi realizado com o indivíduo na posição sentada, com clipe nasal, onde ele realizou uma inspiração máxima até o nível da Capacidade Pulmonar Total (CTP) e, em seguida realizou uma expiração forçada, rápida e completa (Capacidade Vital Forçada - CVF), com avaliação de Volume Expiratório Forçado no Primeiro segundo (VEF1), Capacidade vital forçada (CVF), Pico de Fluxo Expiratório (PFE) ${ }^{9}$.

Foi realizado a manovacuometria para avaliar a Pressão Inspiratória Máxima (PImáx) e a Pressão Expiratória Máxima (PEmáx). Esse procedimento também foi realizado com o paciente em posição sentada, pés apoiados no chão e com clipe nasal ${ }^{10}$. Primeiro solicitou uma inspiração máxima partindo do Volume Residual (VR) com a boca acoplada no bucal do aparelho, depois realizou uma inspiração profunda e em seguida orientado a expirar todo o ar no bucal do aparelho. O procedimento foi realizado por 3 vezes e foi escolhido o melhor resultado para determinação dos valores. As equações utilizadas foram: para homens: PImáx: $\mathrm{y}=-0,80 \times$ idade $+155,3$, PEmáx: $y=-0,81 \times$ idade $+165,3$, Já para as mulheres: PImáx: $y=-0,49 \times$ idade + 110,4 e PEmáx: $y=-0,61 \times$ idade $+115,6^{10}$. 
Os dados obtidos foram digitados em planilha do programa Microsoft Excel 2010 e analisados estatisticamente com o auxílio do Software Statistica 8.0. Foram utilizadas tabelas de frequências com percentual simples e de dupla entrada para as variáveis qualitativas. Foi utilizado à média, desvio padrão mínimo e máximo para as variáveis quantitativas. Também foi utilizado o teste Exato de Fisher para verificação de associação entre as variáveis. O nível de significância adotado no teste foi de $5 \%$, ou seja, foram consideradas significativas as associações cujo $p<0,05$.

\section{Resultados}

Foram entrevistados 15 homens com idade média de 29,9 96,0 anos, cujo IMC médio foi de $25,0 \pm 3,1$. A idade média de início do uso de drogas foi de 18,9 $\pm 4,2$ anos. O tempo médio de uso de drogas foi de $9,4 \pm 6,7$ anos (Tabela 1).

Tabela 1: Distribuição da média, desvio padrão, mínimo e móximo das varióveis avaliadas nos entrevistados

\begin{tabular}{ccccccc}
\hline Variáveis & $\mathrm{n}$ & Média & $\begin{array}{c}\text { Desvio } \\
\text { Padrão }\end{array}$ & Mínimo & Máximo \\
\hline Idade & 15 & 29,9 & \pm & 6,0 & 18,0 & 42,0 \\
\hline IMC & 15 & 25,0 & \pm & 3,1 & 19,1 & 30,2 \\
\hline $\begin{array}{l}\text { Inicio do } \\
\text { uso de } \\
\text { drogas }\end{array}$ & 15 & 18,9 & \pm & 4,2 & 12,0 & 25,0 \\
\hline $\begin{array}{l}\text { Tempo } \\
\text { de uso de } \\
\text { drogas }\end{array}$ & 9,4 & \pm & 6,7 & 1,0 & 25,0 \\
\hline $\begin{array}{l}\text { IMC: índice de massa corporal. } \\
\text { Fonte: os autores. }\end{array}$ & & & & & \\
\hline
\end{tabular}

A maioria dos entrevistados, $60,0 \%(\mathrm{n}=9)$ não teve distúrbio ventilatório. $13,3 \%(\mathrm{n}=2)$ tiveram CVF adequado, 40,0\% ( $n=6)$ VEF 1 adequado e o mesmo percentual teve IT adequado. 26,7\% ( $n=4)$ dos homens tiveram PFE adequado, $66,7 \%$ (n=10) tiveram PImáx adequado o mesmo percentual teve PEmáx também adequado. A maioria dos entrevistados, $66,7 \%(n=10)$ afirmou que tiveram o primeiro contato com drogas por meio de amigos. 53,3\% $(n=8)$ tinham 30 anos ou mais. $46,7 \%(n=7)$ têm peso normal e o mesmo percentual pré - obesidade (Tabela 2).

Tabela 2: Distribuição de frequência das varióveis avaliadas nos entrevistados

\begin{tabular}{|c|c|c|}
\hline Variáveis & $\mathrm{n}$ & $\%$ \\
\hline \multicolumn{3}{|l|}{ Resultado espirometria } \\
\hline Sem distúrbio Ventilatório & 9 & 60,0 \\
\hline Distúrbio ventilatório restritivo leve & 1 & 6,7 \\
\hline Distúrbio ventilatório obstrutivo leve & 3 & 20,0 \\
\hline Distúrbio ventilatório obstrutivo grave & 2 & 13,3 \\
\hline \multicolumn{3}{|l|}{ CVF } \\
\hline Inadequado & 13 & 86,7 \\
\hline Adequado & 2 & 13,3 \\
\hline \multicolumn{3}{|l|}{ VEF1 } \\
\hline Inadequado & 9 & 60,0 \\
\hline Adequado & 6 & 40,0 \\
\hline
\end{tabular}

PFE

\begin{tabular}{ccc} 
Inadequado & 11 & 73,3 \\
Adequado & 4 & 26,7 \\
\hline
\end{tabular}

PI máx

PE máx

Inadequado $\quad 5 \quad 33,3$

Adequado

1066,7

\begin{tabular}{ccc} 
Inadequado & 5 & 33,3 \\
Adequado & 10 & 66,7 \\
\hline Como teve o primeiro contato com drogas & \\
Amigos e balada & 3 & 20,0 \\
Prostituição & 1 & 6,7 \\
Amigos & 10 & 66,7 \\
Amigos e internet & 1 & 6,7 \\
\hline Faixa etária & & \\
De 18 a 29 anos & 7 & 46,7 \\
30 anos ou mais & 8 & 53,3 \\
\hline
\end{tabular}

CVF: capacidade vital forçada, VEF1: volume expiratório forçado no primeiro segundo;

PFE: pico de fluxo expiratório, PImax: pressão inspiratória máxima, PEmax: pressão expiratória máxima. Fonte: os autores.

A maior parte dos homens, $86,7 \% \quad(n=13)$ afirmou ter usado maconha, 53,3\% ( $n=8), 20,0 \%$ $(n=3)$ usaram heroína, nenhum deles usou ecstasy, 6,7\% (n=1) afirmou e usado Dietilamida do Ácidoo Lisérgico (LSD) e o mesmo percentual tiner ou cola. $40,0 \%(n=6)$ estavam de 4 a 11 me- 
ses sem utilizar drogas e 53,3\% ( $n=8)$ utilizam drogas todos os dias. $93,3 \%(n=14)$ consumiam bebida alcoólica anteriormente e 46,7\% ( $n=7)$ tinham habito de consumir duas ou mais vezes ao dia. Todos os entrevistados afirmaram que anteriormente usavam cigarro e 40,0\% ( $n=6)$ afirmaram que fumavam um maço por dia. 33,3\% (n=5) já fizeram tratamentos para parar de consumir drogas, $20 \%(n=3)$ deles fizeram menos de quatro tratamentos (Tabela 3).

A maior parte dos homens, $86,7 \%(n=13)$ afirmou que fuma, 53,3\% ( $\mathrm{n}=8), 46,7 \%(\mathrm{n}=7)$ menos de um maço por dia, $66,7 \%(n=10)$ apresenta falta de ar atualmente e 40,0\% 9n6) quando faz pequenos esforços. Pouco menos da metade, $46,7 \%(\mathrm{n}=7)$ atualmente apresente tosse, $26,7 \%$ $(n=4)$ tosse seca e 20,0\% (n=3) com secreção. 60\% $(n=9)$ apresentam atualmente expectoração de catarro e $46,7 \%(n=7)$ de aspecto escuro. Somente uma pessoa $(6,7 \%)$ afirmou ter corrimento ou secreção nasal de cor amarela. E um homem também apresentou dor torácica $(6,7 \%)$ ao respirar. Não ficou evidenciada associação estatisticamente significativa entre Espirometria e as variáveis avaliadas (Tabela 4).

\section{Discussão}

O presente estudo teve por finalidade caracterizar o perfil da população em tratamento no CAPS/AD para o uso do crack na cidade de Maringá e levantar informações sobre a função pulmonar e força muscular respiratória desses indivíduos. Verificou-se na amostra estudada, que a maioria não apresentou distúrbio ventilatório.

As literaturas ainda não definiram com clareza os efeitos relacionados entre o uso do crack e o sistema respiratório, mas o que se sabe é que as alterações são causadas por ação direta da droga e outras substâncias que são inaladas juntamente com a mesma causam alterações pulmonares, morfológicas e variadas patologias como, a hemossiderose, antracnose, necrose pulmonar, entre outras, ${ }^{71-15}$.
Tabela 3: Distribuição de frequência das varióveis relacionadas as drogas avaliadas nos entrevistados

\begin{tabular}{|c|c|c|}
\hline Variáveis & $\mathrm{n}$ & $\%$ \\
\hline \multicolumn{3}{|l|}{ Usou maconha } \\
\hline Sim & 13 & 86,7 \\
\hline Não & 2 & 13,3 \\
\hline \multicolumn{3}{|l|}{ Usou cocaína } \\
\hline Sim & 8 & 53,3 \\
\hline Não & 7 & 46,7 \\
\hline \multicolumn{3}{|l|}{ Usou heroína } \\
\hline Não & 12 & 80,0 \\
\hline Sim & 3 & 20,0 \\
\hline \multicolumn{3}{|l|}{ Usou Ecstasy } \\
\hline Não & 15 & 100,0 \\
\hline \multicolumn{3}{|l|}{ Usou LSD } \\
\hline Não & 14 & 93,3 \\
\hline Sim & 1 & 6,7 \\
\hline \multicolumn{3}{|l|}{ Usou outra droga } \\
\hline Não & 14 & 93,3 \\
\hline Sim (Tiner cola) & 1 & 6,7 \\
\hline \multicolumn{3}{|l|}{ Tempo sem drogas } \\
\hline 3 meses ou menos & 4 & 26,7 \\
\hline 4 meses a 11 meses & 6 & 40,0 \\
\hline De 1 a 5 anos & 3 & 20,0 \\
\hline Mais de 6 anos & 2 & 13,3 \\
\hline
\end{tabular}

Frequência de uso de drogas

$\begin{array}{lll}\text { Finais de semana } & 1 & 6,7\end{array}$

2 ou 3 vezes na semana $\quad 2 \quad 13,3$

4 ou 5 vezes por semana $\quad 2 \quad 13,3$

6 ou 7 dias na semana $\quad 2 \quad 13,3$

Todos os dias ou quase todos os dias $8 \quad 53,3$

Uso de bebida alcoólica (anterior)

\begin{tabular}{ccc} 
Sim & 14 & 93,3 \\
Não & 1 & 6,7 \\
\hline Uso de bebida alcoólica (anterior) & & \\
1 vez ao dia & 2 & 13,3 \\
2 ou mais vezes por dia & 7 & 46,7 \\
1 vez por semana & 3 & 20,0 \\
2 ou mais vezes por semana & 2 & 13,3 \\
Não consumia & 1 & 6,7 \\
\hline Uso de cigarro (anterior) & & \\
Sim & 15 & 100,0 \\
\hline Frequência de uso de cigarros & & \\
Menos de um maço & 4 & 26,7 \\
1 maço por dia & 6 & 40,0 \\
2 ou mais maços por dia & 5 & 33,3 \\
\hline
\end{tabular}

LSD: dietilamida do ácido lisérgico.

Fonte: os autores. 
Tabela 4: Distribuição de frequência das varióveis segundo resultado da espirometria nos entrevistados

\begin{tabular}{|c|c|c|c|c|c|c|c|c|c|}
\hline \multirow{3}{*}{ Variáveis } & \multicolumn{8}{|c|}{ Espirometria - Resultado } & \multirow{3}{*}{$p^{*}$} \\
\hline & \multicolumn{2}{|c|}{$\begin{array}{l}\text { Sem distúrbio } \\
\text { Ventilatório }\end{array}$} & \multicolumn{2}{|c|}{$\begin{array}{l}\text { Distúrbio ventilatório } \\
\text { restritivo leve }\end{array}$} & \multicolumn{2}{|c|}{$\begin{array}{c}\text { Distúrbio ventilatório } \\
\text { obstrutivo leve }\end{array}$} & \multicolumn{2}{|c|}{$\begin{array}{l}\text { Distúrbio ventilatório } \\
\text { obstrutivo grave }\end{array}$} & \\
\hline & $\mathrm{n}$ & $\%$ & $\mathrm{n}$ & $\%$ & $\mathrm{n}$ & $\%$ & $\mathrm{n}$ & $\%$ & \\
\hline \multicolumn{10}{|l|}{ CVF } \\
\hline Inadequado & 7 & 46,7 & 1 & 6,7 & 3 & 20,0 & 2 & 13,3 & 0,67342 \\
\hline Adequado & 2 & 13,3 & 0 & 0,0 & 0 & 0,0 & 0 & 0,0 & \\
\hline \multicolumn{10}{|l|}{ VEF1 } \\
\hline Inadequado & 3 & 20,0 & 1 & 6,7 & 3 & 20,0 & 2 & 13,3 & 0,08332 \\
\hline Adequado & 6 & 40,0 & 0 & 0,0 & 0 & 0,0 & 0 & 0,0 & \\
\hline \multicolumn{10}{|l|}{ PFE } \\
\hline Inadequado & 5 & 33,3 & 1 & 6,7 & 3 & 20,0 & 2 & 13,3 & 0,30351 \\
\hline Adequado & 4 & 26,7 & 0 & 0,0 & 0 & 0,0 & 0 & 0,0 & \\
\hline \multicolumn{10}{|l|}{ PI máx } \\
\hline Inadequado & 3 & 20,0 & 0 & 0,0 & 0 & 0,0 & 2 & 13,3 & 0,11162 \\
\hline Adequado & 6 & 40,0 & 1 & 6,7 & 3 & 20,0 & 0 & 0,0 & \\
\hline \multicolumn{10}{|l|}{ PE máx } \\
\hline Inadequado & 3 & 20,0 & 0 & 0,0 & 0 & 0,0 & 2 & 13,3 & 0,11162 \\
\hline Adequado & 6 & 40,0 & 1 & 6,7 & 3 & 20,0 & 0 & 0,0 & \\
\hline \multicolumn{10}{|c|}{$\begin{array}{l}\text { CVF: capacidade vital forçada, VEF1: volume expiratório forçado no primeiro segundo; } \\
\text { PFE: pico de fluxo expiratório, PImax: pressão inspiratória máxima, PEmax: pressão expiratória máxima. } \\
\text { * p não significativo pelo teste Exato de Fisher considerando nível de significância de } 5 \%\end{array}$} \\
\hline Fonte: os autores. & & & & & & & & & \\
\hline
\end{tabular}

Os achados com relação à função pulmonar de usuários de crack, ainda são controversos. Em um estudo ${ }^{16}$ avaliaram a função pulmonar com 28 indivíduos antes e após o tratamento ao uso de drogas, os resultados apresentaram normais, porém após 60 dias de uso da droga, houve significativos decréscimos no VEF1, porém essas reduções ainda mantiveram dentro dos parâmetros da normalidade. Resultados semelhantes foram encontrados no presente estudo, em que a maioria não apresentou distúrbios ventilatórios.

A maioria dos estudos tem enfatizado as complicações pulmonares devido ao uso de drogas ilícitas. A administração tanto por via endovenosa, quanto inalatória ocasiona diversas alterações morfológicas no sistema respiratório. Essas alterações estão relacionadas com a frequência e o tempo de utilização, bem como à via de administração ${ }^{17}$.
Em um estudo ${ }^{18}$ acompanharam o efeito do uso de drogas ilícitas no período de oito anos. Ao final, evidenciaram um declínio do Índice de Tiffeneau. Os autores enfatizaram que o uso das drogas ilícitas afeta negativamente a função pulmonar dos usuários.

Vários fatores contribuem para a diversidade dos achados nos estudos, entre eles, destacam-se o tamanho amostral, uso de outras substâncias, ausência de grupo controle e quantidade de substâncias consumida, além do padrão de tabagismo e profundidade de inalação ${ }^{19}$. Mesmo os achados relacionados às alterações da capacidade pulmonar ainda são inconclusivos, alguns achados morfológicos no sistema respiratório estão mais esclarecidos. Em estudos laboratoriais com ratos, foi demonstrado que a exposição à fumaça da maconha é capaz de promover inflamação nas vias aéreas ${ }^{20}$. 
No estudo ${ }^{20}$ em que investigaram a função pulmonar e a capacidade funcional de dependentes químicos internados em um centro de reabilitação, após avaliação dos participantes não apresentaram alterações em ambas variáveis estudadas. Resultados semelhantes foram encontrados no presente estudo, onde a maioria da amostra não foi identificada distúrbios ventilatórios.

No presente estudo nota-se que anteriormente ao tratamento os indivíduos não apresentaram tosse, expectoração e dor torácica, porém atualmente durante o tratamento observam-se a presença de expectoração de cor escura. Como os pulmões são os primeiros órgãos que estão expostos aos produtos de combustão do crack, os sintomas agudos respiratórios podem desenvolver-se dentro de minutos ou várias horas após o seu uso, sendo que as principais queixas respiratórias são tosse, expectoração, dor torácica e hemoptíase ${ }^{21}$.

O uso de crack está apontado como responsável por $35 \%$ do consumo total de drogas ilícitas no país. Atualmente o crack tornou-se um problema em saúde pública e tem demonstrado seu poder de promover desfechos em saúde com graves consequências ${ }^{7,22}$.

Durante as buscas, o presente estudo é um dos poucos que investigaram a função pulmonar e força muscular respiratória de dependentes químicos em processo de tratamento. A investigação desses aspectos nessa população é de suma importância para compreensão da natureza dos distúrbios decorrentes do uso de substancias psicoativas ilícitas, bem como para a determinação de políticas e ações de promoção da saúde dirigidas a esta condição ${ }^{20}$.

As limitações do presente estudo podem ser destacadas como pequeno tamanho amostral e o fato de que os indivíduos incluídos possuíam um tempo de utilização de substancias psicoativas inferior a vinte anos, isso pode ter colaborado para que não fossem encontradas relevantes alterações na função pulmonar.

\section{Conclusões}

Apesar de não existir muitos trabalhos relacionados ao uso do crack, sabe-se que é uma droga já bastante conhecida pela população e o seu consumo leva a uma rápida dependência, pelos seus efeitos prazerosos e por seu tempo de duração ser de curto prazo. Os sinais e sintomas relatados pelos usuários servem de alerta para a população para o quanto essa droga pode gerar de malefícios para os indivíduos tanto no aspecto pessoal como social. Porém os resultados apresentados indicam que a função pulmonar e a força muscular respiratória realizada com a população em tratamento no CAPS/AD para o uso de crack na cidade de Maringá-PR não apresentaram alterações significativas. Sugere-se que sejam realizadas, novas pesquisas com um maior tamanho amostral, pessoas do sexo feminino e grupo controle.

\section{Referências}

1. Carlini EA, Nappo SA, Galduróz JCF, Noto AR. Drogas psicotrópicas: o que são e como agem. Revista Imesc. 2001;3(3):9-35.

2. Costa MRSR, Alves RF, Franca MD. Manifestações pulmonares causadas pelo uso do crack. Jornal Brasileiro de Pneumologia. 1998;24(5):317-321.

3. Mançano A, Marchiori E, Zanetti G, Escuissato DL, Duarte BC, Apolinário LA. Complicações pulmonares após uso de crack: achados na tomografia computadorizada de alta resolução do tórax. Jornal Brasileiro de Pneumologia. 2008;34(5):323-327.

4. Etchepare M, Dotto ER, Domingues KA, Colpo E. Perfil de adolescentes usuários de crack e suas consequência metabólicas. Revista da Amrigs. 2011;55(2):140-146.

5. Muakad IB. A cocaína e o crack: as drogas da morte. Revista da Faculdade de Direito da Universidade de São Paulo. 2011;106(1):465-494. Disponível em: http://www.producao.usp.br/bitstream/handle/ BDPI/43703/70562.pdf?sequence $=1$ 
6. Kelter PJ, Silva NTRC. Legalidade e Finalidade da Internação Compulsória dos Dependentes de Crack. Revista Jurídica Cesumar. 2013;13(2):45-53.

7. Horta RL, Schafer JL, Coelho LRM, Rodrigues VS, Oliveira MS, Teixeira VA. Conditions associated with impaired social skills in a convenience sample of crack users. Cadernos de Saúde Pública. 2016;32(4):15-28.

8. Oliveira LG, Nappo SA. Crack na cidade de São Paulo: acessibilidade, estratégias de mercado e formas de uso. Archives of Clinical Psychiatry. 2008;35(6):212-218.

9. Presto B, Damázio L. Fisioterapia na UTI. 2. ed. Rio de Janeiro: Elsevier, 2009.

10. Neder JA, Andreoni S, Lerario MC, Nery LE. Reference values for lung function tests. II. Maximal respiratory pressures and voluntary ventilation. Brazilian Journal of Medical and Biological Research. 1999;32(6):719-27.

11. Costa Júnior AF, Baldaçara LR, Silva AS, Tavares ACFR, Orsolin EF, Prehl VB, et al. Profile of peripheral vascular changes in crack-cocaine addicts receiving treatment at a Psychosocial Care Center for Alcohol and Drugs. Jornal Vascular Brasileiro. 2016;15(2):126-133.

12. Rocha WS, Alves ERP, Vieira KFL, Barbosa KKS, Leite GO, Dias MD. Concepções dos usuários de crack sobre os fatores que influenciam o uso ea dependência. Revista Eletrônica Saúde Mental Álcool e Drogas. 2015;11(3):129-135.

13. Teixeira AA, Kantrski LP, Corrêa ACL, Ferreira RZ, Ferreira GB, Santo MOE. Usuários de crack: desenvolvendo estratégias para enfrentar os riscos do uso. Revista de Pesquisa Cuidado é Fundamental. 2015;7(2):2393-2404
14. Bulbena-Cabre A, Dunn NR, Swift RG. "Crack Lung": A Pulmonary Complication of Crack Cocaine Case. Journal of Addiction Medicine. 2015;9(2):164165.

15. Shah R, Patel A, Mousa O, Manocha D. Crack lung: cocaine-induced lung injury. International Journal of Medicine. 2015;108(9):749-759.

16. Tashkin DP, Shapiro BJ, Lee YE, Harper CE. Subacute effects of heavy marihuana smoking on pulmonary function in healthy men. New England Journal of Medicine. 1976;294(3):125-129.

17. Glassroth J, Adams GD, Schnoll S. The impact of substance abuse on the respiratory system. CHEST Journal. 1987;91(4):596-602.

18. Taylor DR, Fergusson DM, Milne BJ, Horwood LJ, Moffitt TE, Sears MR, et al. A longitudinal study of the effects of tobacco and cannabis exposure on lung function in young adults. Addiction. 2002;97(8):1055-1061.

19. Haim DY, Lippmann ML, Goldberg SK, Walkenstein MD. The pulmonary complications of crack cocaine: a comprehensive review. CHEST Journal. 1995;107(1):233-240

20. Seleghim MR, Oliveira MLF. Influência do ambiente familiar no consumo de crack em usuários. Acta Paulista de Enfermagem. 2013;26(3):263-628.

21. Andrade AT, Rimes TS, Csta LSP, Jorge MSB, Quinderé PHD. Social and demographical aspects of crack users assisted by psychological-social attention network. Revista Eletrônica Saúde Mental Álcool e Drogas. 2016;12(1):40-47.

22. Schenker M, Minayo MCS. A implicação da família no uso abusivo de drogas: uma revisão crítica. Ciência \& Saúde Coletiva. 2003;8(1):299-306. 